\title{
Schönheit im Gehirn
}

\section{Erhard Taverna}

Dr. med., Mitglied der Redaktion

In den drei vorgestellten Werken geht es im weitesten Sinne um «Schönheit im Gehirn»: Die Autoren beeindrucken mit wissenschaftlich fundierten Kenntnissen über die Verarbeitung von Wahrnehmungen, mit einem feinsinnigen Verständnis für die menschliche Seele oder mit einem praktischen Leitfaden zu Anatomie und Physiologie, der sich an Fachpersonen aus dem Gesundheitswesen richtet.

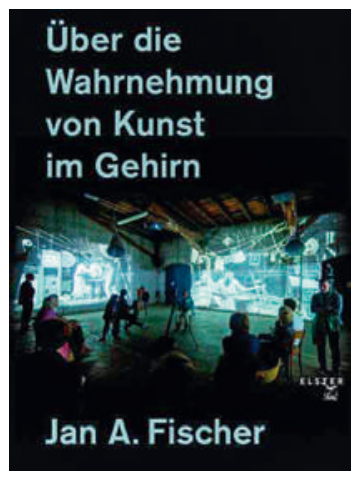

Jan A. Fischer

Über die Wahrnehmung von Kunst im Gehirn

Zürich: Elster Verlag; 2016.

168 Seiten. 39.80 CHF.

ISBN 978-3-906-06542-7

Jan A. Fischers drittes Buch handelt von der Wahrnehmung von Kunst im Gehirn. Im ersten Buch ging es um seinen Weg zum Kunstsammler, im zweiten um Begegnungen mit lateinamerikanischen und asiatischen Künstlern. Jetzt hat auch die Neurowissenschaft ihren Auftritt. Im ersten Teil erklärt Fischer die Architektur des Nervensystems und Untersuchungsmethoden, wie funktionelle Magnetresonanz fMRI, PositronenEmissionstomographie, PET und Psychologie. Dem Sehvorgang kommt bei der bildenden Kunst die grösste Bedeutung zu. Der Autor stützt sich dabei häufig auf die Ergebnisse des britischen Neurobiologen Semir Zeki, der auch Kunstsammler und Maler ist und die Areale V1 bis V6 der visuellen Wahrnehmung im Gehirn bei vielen Probanden untersucht hat. Je mehr Kunst gefiel, desto stärker wurde der mediale orbito-frontale Kortex aktiviert. Wie immer bei Emotionen spielen auch neuronale Erinnerungs- und Belohnungszentren eine Rolle. Ein komplexer Vorgang, den Fischer in gedrängter, aber gut verständlicher Form mit zahlreichen Bildbeispielen verdeutlicht. Zeki sucht eine universelle Signatur des Schönen (und Hässlichen) im Gehirn, von ihm stammt der Begriff der Neuroästhetik. Der zweite Buchteil führt reich bebildert durch die Kunstgeschichte, wobei auch klar wird, dass die Hirnforschung der Kunst mehr verdankt als umgekehrt. Kunsterzeugnisse sind kulturspezifisch, die Beurteilung der Qualität ist zeitbedingt. Hier spricht der Mediziner und Kunstkenner, der seine eigene Rolle als Betrachter selbstkritisch hinterfragt. Die neuen Erkenntnisse sind eine Bereicherung, wie jeder neue Blickwinkel auf Werke der bildenden Kunst, denn es gibt viele Wege, das Gehirn anzuregen. Immer sind kontextabhängig alle Sinne an der Beurteilung eines Kunstwerkes beteiligt. Jan A. Fischer hat ein Werk vorgelegt, das die linke und die rechte Hemisphäre gleichermassen beansprucht. Eine kenntnisreiche, unterhaltende Reise durch das Universum der Neurobiologie und der modernen Kunstbetrachtung.

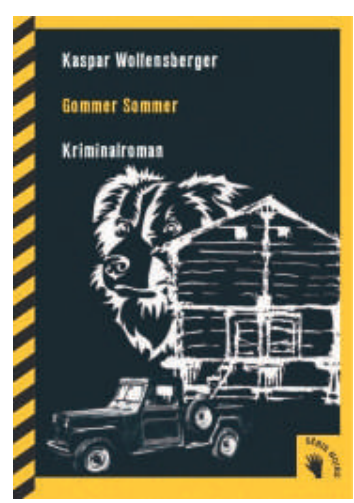

Kaspar Wolfensberger Gommer Sommer

Kriminalroman Zürich: bilgerverlag; 2016. 380 Seiten. 34 CHF. ISBN 978-3-037-62056-4 
Kaspar Wolfensberger, Psychiater und Psychotherapeut in Zürich, hat nach Zanggers Seminar, Glanzmann, Liebeskrank und Der Seelenwexler erneut die Szenerie gewechselt. Der Gommer Sommer ist ein spannender Kriminalroman, in dem ein entlassener Dienstchef der Kripo Zürich sich in Münster erholen will, dabei einen erhängten Vermieter vorfindet und gegen alle Vorsätze in einer üblen Geschichte zu ermitteln beginnt. Einen Roman, der von Geheimnissen und Überraschungen lebt, soll man nicht nacherzählen. Land und Leute, Filz und Bauwirtschaft sind die Zutaten zu einem Puzzle, das der «Üsserschwiizer» Schnüffler mit Hilfe der Walliser Kollegen Stück für Stück zum finalen Schlussbild zusammensetzt. Ein seltsamer Kauz, der auch so heisst, hartnäckig und begabt, einsam und dennoch empathisch. Man muss ihn mögen, wenn man an seinen Begegnungen mit Einheimischen teilnimmt und ihn auf seinen vielen Wanderungen durch das Obergoms begleitet. Die Erzählung lebt vom Lokalkolorit, das die Kennerschaft des Autors verrät. Doch fern von Klischees und Heimatkitsch entwirft Wolfensberger ein weites Panorama alpenländischer Zustände, die man auf die ganze Schweiz übertragen kann. Ohne jemals an Schwung und Rhythmus zu verlieren, findet eine dramatische Geschichte ihr logisches Ende. Weil sie einen ganz eigenen Sog entwickelt, ist es schwierig, mit dem Lesen aufzuhören. Bleibt abzuwarten, wer als Erster den Filmstoff darin entdeckt.

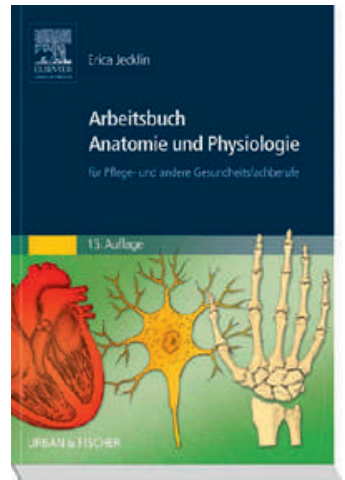

Erica Jecklin

\section{Arbeitsbuch Anatomie und Physiologie}

München: Urban \& Fischer Verlag; 2016.

416 Seiten. 42.90 CHF.

ISBN 978-3-437-26983-7

Ein Buch, das in der 15. Auflage vorliegt und schon fast eine halbe Million Mal verkauft wurde, muss als geschätztes Standardwerk nicht mehr vorgestellt werden. Erica Brühlmann-Jecklin hat 1976 die Lehrerinnenausbildung für Krankenpflege abgeschlossen und danach während zwei Semestern an der medizinischen Fakultät der Universität Zürich die Anatomievorlesungen von Professor Kubik besucht. Von der ersten Auflage 1980 bis zur siebten Auflage hat die Autorin alle Zeichnungen selber von Hand angefertigt, erst danach wurden sie von einer Computerzeichnerin als Vorlagen übernommen. Der Inhalt ist bis heute konsequent nach Topographie, Makro-, Mikroskopie und Physiologie gegliedert und war damals das einzige Arbeitsbuch, das am Ende eines jeden Kapitels Fragen stellte, die mit Hilfe von Seitenangaben das Selbststudium vertieften. Viele Medizinstudenten benutzten das Werk zur Vorbereitung des zweiten Propädeutikums. Die erweiterte und aktualisierte Neuauflage richtet sich an Pflege- und andere Gesundheitsfachberufe, leistet aber auch in der ärztlichen Praxis, dank ausführlichem Register, als kurze Einführung und Übersicht praktische Dienste. Der Gründerin, der seit 1974 in Zürich etablierten "Gesellschaft für Muskelkranke», ist einmal mehr eine didaktisch vorbildliche Arbeit gelungen, die auch in medizinischen Kreisen eine Würdigung verdient. 